\title{
POINTWISE MULTIPLIERS FOR CAMPANATO SPACES ON GAUSS MEASURE SPACES
}

\author{
LIGUANG LIU AND DACHUN YANG
}

\begin{abstract}
In this paper, the authors characterize pointwise multipliers for Campanato spaces on the Gauss measure space $\left(\mathbb{R}^{n},|\cdot|, \gamma\right)$, which includes $\operatorname{BMO}(\gamma)$ as a special case. As applications, several examples of the pointwise multipliers are given. Also, the authors give an example of a nonnegative function in $\operatorname{BMO}(\gamma)$ but not in $\operatorname{BLO}(\gamma)$.
\end{abstract}

\section{$\S 1$. Introduction}

The pioneering work on the characterizations of pointwise multipliers on $\mathrm{BMO}_{\phi}\left(\mathbb{T}^{n}\right)$ and the Hardy space $H^{1}\left(\mathbb{T}^{n}\right)$ was due to Janson [14], where $\mathbb{T}^{n}$ is the $n$-dimensional torus and $\mathrm{BMO}_{\phi}\left(\mathbb{T}^{n}\right)$ is the function space defined by using the mean oscillation and some growth function $\phi$. Specifically, when $n=1$, the same characterizations as in [14] of the pointwise multipliers on $\operatorname{BMO}(\mathbb{T})$ and $H^{1}(\mathbb{T})$ have been obtained by Stegenga [37], which were further used to study the boundedness of the Toeplitz operator on $H^{1}(\mathbb{T})$. Later, Nakai and Yabuta [30] extended Janson's results to the $n$-dimensional Euclidean space $\mathbb{R}^{n}$. In particular, they proved that $g$ is a pointwise multiplier on $\operatorname{BMO}\left(\mathbb{R}^{n}\right)$ (viz., the multiplication $g f \in \operatorname{BMO}\left(\mathbb{R}^{n}\right)$ for all $f \in$ $\left.\operatorname{BMO}\left(\mathbb{R}^{n}\right)\right)$ if and only if $g \in L^{\infty}\left(\mathbb{R}^{n}\right) \cap \mathrm{BMO}^{\log }\left(\mathbb{R}^{n}\right)$, where $g \in \mathrm{BMO}^{\log }\left(\mathbb{R}^{n}\right)$ means that $g \in L_{\mathrm{loc}}^{1}\left(\mathbb{R}^{n}\right)$ and that

Received August 4, 2012. Revised February 13, 2013. Accepted May 14, 2013.

First published online March 25, 2014.

2010 Mathematics Subject Classification. Primary 42B15; Secondary 42B30, 28 C20.

Liu's work was partially supported by National Natural Science Foundation of China grant 11101425. Corresponding author Dachun Yang's work was partially supported by National Natural Science Foundation of China grants 11171027 and 11361020, the Specialized Research Fund for the Doctoral Program of Higher Education of China grant 20120003110003, and the Fundamental Research Funds for the Central Universities of China grant 2012LYB26.

(C) 2014 by The Editorial Board of the Nagoya Mathematical Journal 


$$
\begin{aligned}
& \|g\|_{\mathrm{BMO}^{\log }\left(\mathbb{R}^{n}\right)} \\
& \quad:=\sup _{B \subset \mathbb{R}^{n}} \frac{\left|\log r_{B}\right|+\log \left(e+\left|c_{B}\right|\right)}{|B|} \int_{B}\left|g(x)-\frac{1}{|B|} \int_{B} g(z) d z\right| d x<\infty,
\end{aligned}
$$

where $c_{B}$ and $r_{B}$ represent, respectively, the center and the radius of the ball $B$ and where the supremum is taken over all balls of $\mathbb{R}^{n}$. Observe that, as $\operatorname{BMO}\left(\mathbb{R}^{n}\right)$ is a translation-invariant space but $\mathrm{BMO}^{\log }\left(\mathbb{R}^{n}\right)$ is not, the multipliers considered in [30] are not assumed to be norm bounded. In [31], the results of [30] were also generalized to spaces of homogeneous type in the sense of Coifman and Weiss (see [3], [4]). (For the characterization of pointwise multipliers on other function spaces such as Lorentz and OrliczLebesgue spaces, we refer the reader to [22], [27]-[29], [39], [13], and their references.) It should be mentioned that the class of pointwise multipliers for $\mathrm{BMO}\left(\mathbb{R}^{n}\right)$ has been used by Lerner [16] to solve a conjecture of Diening [5] on the boundedness of the Hardy-Littlewood maximal operators on the variable Lebesgue spaces. Also, by invoking the results of [30] and [31] on pointwise multipliers, Lin, Nakai, and Yang in [17] constructed a nonnegative function in $\operatorname{BMO}\left(\mathbb{R}^{n}\right)$ but not in $\operatorname{BLO}\left(\mathbb{R}^{n}\right)$, which shows that the results obtained in [17] on the boundedness of the Lusin area and the $g_{\lambda}^{*}$ functions indeed improve the known corresponding results even on $\mathbb{R}^{n}$. Recently, Ky [15] further proved that the space BMO ${ }^{\log }\left(\mathbb{R}^{n}\right)$ is the dual of some Musielak-Orlicz Hardy space $H^{\log }\left(\mathbb{R}^{n}\right)$.

The main aim of this article is to study characterizations of pointwise multipliers for Campanato spaces on the Gauss measure space, namely, the Euclidean space $\mathbb{R}^{n}$ endowed with the Euclidean distance $|\cdot|$ and the Gauss measure $\gamma$, where, for all $x \in \mathbb{R}^{n}$,

$$
d \gamma(x):=\pi^{-n / 2} e^{-|x|^{2}}
$$

Notice that the Gauss measure space is not a space of homogeneous type (see [3], [4]); namely, there does not exist a positive constant $C$ such that $\gamma(2 B) \leq$ $C \gamma(B)$ for all open balls $B$ in $\mathbb{R}^{n}$. From a viewpoint of self-adjointness, we see that $\gamma$ is a natural measure associated to the Ornstein-Uhlenbeck operator $\mathcal{L}:=-\Delta / 2+x \cdot \nabla$. The operator $\mathcal{L}$ behaves like the "Laplacian" on the Gauss measure space. But the kernels of singular integrals associated with $\mathcal{L}$, such as Riesz transforms, satisfy the standard Calderón-Zygmund kernel estimates

$$
|K(x, y)| \leq \frac{C_{\eta}}{|x-y|^{n}} \quad \text { and } \quad\left|\partial_{x} K(x, y)\right|+\left|\partial_{y} K(x, y)\right| \leq \frac{C_{\eta}}{|x-y|^{n+1}}
$$


only on the region $\left\{(x, y) \in \mathbb{R}^{n} \times \mathbb{R}^{n}: 0<|x-y|<\eta \min \{1,1 /|x|\}\right\}$ with fixed $\eta \in(0, \infty)$; the positive constant $C_{\eta}$ increases exponentially to infinity as $\eta \rightarrow \infty$. In the last few decades, there has been a huge amount of literature on analysis over the Gauss measure space and the Ornstein-Uhlenbeck operator $\mathcal{L}$, with the emphasis on the boundedness on $L^{p}(\gamma)$ with $p \in[1, \infty)$ of singular integrals associated with $\mathcal{L}$ (see, e.g., [6], [7], [10], [11], [8], [9], [33], [32], [35], [38]) or with the Ornstein-Uhlenbeck semigroup (see, e.g., [12], [26], [34]).

A recent breakthrough on this subject was due to Mauceri and Meda [23], in which the Hardy space $H^{1}(\gamma)$ and its dual space $\operatorname{BMO}(\gamma)$ were introduced and the boundedness of singular integrals associated with $\mathcal{L}$ on these function spaces was established. The theory of [23] relies on the introduction of a class of admissible balls on which the measure $\gamma$ is doubling. To recall the definitions of $\operatorname{BMO}(\gamma)$ and $H^{1}(\gamma)$, we begin with some notation and notions used in [23]. Define $m(x):=\min \{1,1 /|x|\}$ for all $x \in \mathbb{R}^{n}$. For any $a \in(0, \infty)$, denote by $\mathcal{B}_{a}$ the set of all balls $B$ in $\mathbb{R}^{n}$ such that $r_{B} \leq a m\left(c_{B}\right)$, where $c_{B}$ and $r_{B}$ denote the center and the radius of $B$, respectively. Balls in $\mathcal{B}_{a}$ are called admissible balls with scale $a$. If $B \in \mathcal{B}_{a}$ and $x \in B$, then

$$
(a+1)^{-1} m(x) \leq m\left(c_{B}\right) \leq(a+1) m(x)
$$

and

$$
e^{-a^{2}-2 a} \leq e^{\left|c_{B}\right|^{2}-|x|^{2}} \leq e^{2 a}
$$

(see [19, Lemma 2.1], [23, Proposition 2.1]). Consequently, for all $B \in \mathcal{B}_{a}$,

$$
\pi^{-n / 2} e^{-a^{2}-2 a} \leq \frac{\gamma(B)}{e^{-\left|c_{B}\right|^{2} r_{B}^{n}}} \leq \pi^{-n / 2} e^{2 a} .
$$

This inequality implies that $\gamma$ is doubling on the admissible class $\mathcal{B}_{a}$; that is, there exists a positive constant $K$ such that, for all balls $B \in \mathcal{B}_{a}$,

$$
\gamma(2 B) \leq K \gamma(B) \quad \text { (locally doubling property) }
$$

A function $f \in L^{1}(\gamma)$ is said to be in the space $\operatorname{BMO}(\gamma)$ provided that

$$
\|f\|_{\mathrm{BMO}(\gamma)}:=\|f\|_{L^{1}(\gamma)}+\sup _{B \in \mathcal{B}_{a}} \frac{1}{\gamma(B)} \int_{B}\left|f(x)-f_{B}\right| d \gamma(x)<\infty,
$$


where, above and in what follows,

$$
f_{B}:=\frac{1}{\gamma(B)} \int_{B} f(y) d \gamma(y)
$$

It was proved in $[23$, Remark 4.3] that, for all $p \in(1, \infty)$,

$$
\|f\|_{\mathrm{BMO}(\gamma)} \sim\|f\|_{L^{1}(\gamma)}+\sup _{B \in \mathcal{B}_{a}}\left[\frac{1}{\gamma(B)} \int_{B}\left|f(x)-f_{B}\right|^{p} d \gamma(x)\right]^{1 / p}
$$

with implicit constants depending only on $a, p$, and $n$. The predual of $\operatorname{BMO}(\gamma)$ is the atomic Hardy space $H^{1}(\gamma)$ (see [23, Theorem 5.2]). Atoms of $H^{1}(\gamma)$ are either the constant function 1 , or a function $a \in L^{1}(\gamma)$ supported on a ball $B \in \mathcal{B}_{1}$ such that $\|a\|_{L^{\infty}(\gamma)} \leq[\gamma(B)]^{-1}$ and $\int_{B} a(x) d \gamma(x)=0$. Then, the atomic Hardy space $H^{1}(\gamma)$ is defined to be the space of all functions $g \in$ $L^{1}(\gamma)$ that admit decompositions of the form $g=\sum_{k=1}^{\infty} \lambda_{k} a_{k}$, where $\left\{a_{k}\right\}_{k=1}^{\infty}$ are atoms of $H^{1}(\gamma)$ and $\left\{\lambda_{k}\right\}_{k=1}^{\infty} \subset \mathbb{C}$ satisfying that $\sum_{k=1}^{\infty}\left|\lambda_{k}\right|<\infty$. The norm $\|g\|_{H^{1}(\gamma)}$ of $g$ is defined to be the infimum of $\sum_{k=1}^{\infty}\left|\lambda_{k}\right|$ over all decompositions of $g$ as above. (For further investigations related to the $H^{1}(\gamma)$ $\operatorname{BMO}(\gamma)$ theory developed in [23], see [2], [1], [20], [21], [24], [25].)

In [18], the notion of $\operatorname{BMO}(\gamma)$ was extended to the Morrey and Campanato spaces, and the boundedness of singular integrals associated with $\mathcal{L}$ was investigated. Let us recall the notions of Morrey and Campanato spaces on the Gauss measure space in [18], as follows.

Definition 1.1. Let $a \in(0, \infty)$, let $p \in[1, \infty)$, and let $\kappa \in(-\infty, 1]$. Any locally integrable function $f$ is said to be in the space $\mathcal{C}_{\mathcal{B}_{a}}^{p, \kappa}(\gamma)$ provided that

$$
\|f\|_{\mathcal{C}_{\mathcal{B}_{a}(\gamma)}^{p, \kappa}}:=\sup _{B \in \mathcal{B}_{a}}\left\{\frac{1}{[\gamma(B)]^{1-\kappa}} \int_{B}\left|f(x)-f_{B}\right|^{p} d \gamma(x)\right\}^{1 / p}<\infty,
$$

where $f_{B}$ is as in (1.5). Moreover, the Campanato space $\mathcal{L}_{\mathcal{B}_{a}}^{p, \kappa}(\gamma)$ is defined to be the collection of all $f \in L^{1}(\gamma) \cap \mathcal{C}_{\mathcal{B}_{a}}^{p, \kappa}(\gamma)$. For each $f \in \mathcal{L}_{\mathcal{B}_{a}}^{p, \kappa}(\gamma)$, define

$$
\|f\|_{\mathcal{L}_{\mathcal{B}_{a}}^{p, \kappa}(\gamma)}:=\|f\|_{L^{1}(\gamma)}+\|f\|_{\mathcal{C}_{\mathcal{B}_{a}}^{p, \kappa}(\gamma)}
$$

If $a=1$, we write $\mathcal{C}_{\mathcal{B}_{a}}^{p, \kappa}(\gamma)$ and $\mathcal{L}_{\mathcal{B}_{a}}^{p, \kappa}(\gamma)$ simply as $\mathcal{C}^{p, \kappa}(\gamma)$ and $\mathcal{L}^{p, \kappa}(\gamma)$ respectively.

REMARK 1.1. For $a \in(0, \infty)$, instead of $\mathcal{B}_{a}$, we consider the collection of admissible cubes $\mathcal{Q}_{a}$, which consists of cubes $Q$ with sides parallel to 
the axes, the center $c_{Q}$, and the side length $\ell_{Q} \leq a m\left(c_{Q}\right)$. For $p \in[1, \infty)$ and $\kappa \in(-\infty, 1]$, we define the spaces $\mathcal{C}_{\mathcal{Q}_{a}}^{p, \kappa}(\gamma), \mathcal{L}_{\mathcal{Q}_{a}}^{p, \kappa}(\gamma)$ and the (semi)norms $\|\cdot\|_{\mathcal{C}_{\mathcal{B}_{a}}^{p, \kappa}(\gamma)}$ and $\|\cdot\|_{\mathcal{C}_{\mathcal{Q} a}^{p, \kappa}(\gamma)}$ as in Definition 1.1 but with balls $B \in \mathcal{B}_{a}$ therein replaced by cubes $Q \in \mathcal{Q}_{a}$. It is proved in Proposition 2.1 below that the (semi)norms $\|\cdot\|_{\mathcal{C}_{\mathcal{B} a}^{p, \kappa}(\gamma)}$ and $\|\cdot\|_{\mathcal{C}_{\mathcal{Q} a}^{p, \kappa}(\gamma)}$ are equivalent and that they are both independent of the choice of $a$.

In what follows, we write $\mathcal{C}_{\mathcal{B}_{a}}^{p, \kappa}(\gamma)$ or $\mathcal{C}_{\mathcal{Q}_{a}}^{p, \kappa}(\gamma)$ simply as $\mathcal{C}^{p, \kappa}(\gamma)$, and we write $\mathcal{L}_{\mathcal{B}_{a}}^{p, \kappa}(\gamma)$ or $\mathcal{L}_{\mathcal{Q}_{a}}^{p, \kappa}(\gamma)$ simply as $\mathcal{L}^{p, \kappa}(\gamma)$, if it causes no confusion.

Definition 1.2. Let $p \in[1, \infty)$, and let $\kappa \in(-\infty, 1]$. A function $g$ is called a pointwise multiplier on $\mathcal{L}^{p, \kappa}(\gamma)$ if, for all $f \in \mathcal{L}^{p, \kappa}(\gamma)$, the multiplication $f g$ belongs to $\mathcal{L}^{p, \kappa}(\gamma)$ and $\|f g\|_{\mathcal{L}^{p, \kappa}(\gamma)} \leq C\|f\|_{\mathcal{L}^{p, \kappa}(\gamma)}$ for some positive constant $C$ independent of $f$.

Notice that, for $p \in[1, \infty)$ and $\kappa \in(0,1] \cap(0, p)$, it was proved in [18, Theorem 3.7] that the spaces $\mathcal{L}^{p, \kappa}(\gamma)$ and $\mathcal{M}^{p, \kappa}(\gamma)$ coincide with equivalent norms, where the Morrey space $\mathcal{M}^{p, \kappa}(\gamma)$ is defined to be the space of all functions $f \in L^{1}(\gamma)$ satisfying that

$$
\|f\|_{\mathcal{M}^{p, \kappa}(\gamma)}:=\sup _{B \in \mathcal{B}_{1}}\left[\frac{1}{[\gamma(B)]^{1-\kappa}} \int_{B}|f(x)|^{p} d \gamma(x)\right]^{1 / p}<\infty .
$$

For characterizations of pointwise multipliers on $\mathcal{L}^{p, \kappa}(\gamma)$ with $\kappa \in(0,1] \cap$ $(0, p)$, we have the following result, whose proof is given in Section 4 below.

Theorem 1.1. Let $p \in[1, \infty)$, and let $\kappa \in(0,1] \cap(0, p)$. Then, a function $g$ is a pointwise multiplier on $\mathcal{M}^{p, \kappa}(\gamma)$ if and only if $g \in L^{\infty}(\gamma)$. Moreover, the operator norm of $g$, which is denoted by $\|g\|_{\mathcal{M}^{p, \kappa} \rightarrow \mathcal{M}^{p, \kappa}}$, is comparable to $\|g\|_{L^{\infty}(\gamma)}$.

Let $p \in[1, \infty)$. Notice that $\mathcal{L}^{p, 0}(\gamma)$ is exactly the space $\operatorname{BMO}(\gamma)$ introduced in [23, p. 281]. Also, for $\kappa \in[-p / n, 0)$, it is proved in Proposition 2.3 below that $\mathcal{C}^{p, \kappa}(\gamma)=\operatorname{Lip}_{-\kappa / p}(\gamma)$ with equivalent seminorms. Here, for any $\beta \in(0,1 / n]$, the Lipschitz space $\operatorname{Lip}_{\beta}(\gamma)$ is defined to be the collection of all locally integrable functions $f$ such that

$$
\|f\|_{\operatorname{Lip}_{\beta}(\gamma)}:=\sup _{\substack{B \in \mathcal{B}_{1} \\ x, y \in B}} \frac{|f(x)-f(y)|}{[\gamma(B)]^{\beta}}<\infty .
$$

But, when $\kappa \in(-\infty,-p / n)$, the space $\mathcal{C}^{p, \kappa}(\gamma)$ consists only of almost everywhere constant functions (see also Proposition 2.3 below). Thus, it makes 
sense to consider only pointwise multipliers on $\mathcal{L}^{p, \kappa}(\gamma)$ with $\kappa \in[-p / n, 0]$. The main result is as follows.

THEOREM 1.2. Let $p \in[1, \infty)$, and let $\kappa \in[-p / n, 0]$. Then, a function $g$ is a pointwise multiplier on $\mathcal{L}^{p, \kappa}(\gamma)$ if and only if $g \in L^{\infty}(\gamma) \cap \mathcal{L}_{\Psi_{\kappa}}^{p, \kappa}(\gamma)$, where the space $\mathcal{L}_{\Psi_{\kappa}}^{p, \kappa}(\gamma)$ is defined to be the collections of all $g \in L_{\text {loc }}^{p}(\gamma)$ such that

$$
\|g\|_{\mathcal{L}_{\Psi_{\kappa}}^{p, \kappa}(\gamma)}:=\sup _{B \in \mathcal{B}_{1}} \Psi_{\kappa}(B)\left[\frac{1}{\gamma(B)} \int_{B}\left|g(x)-g_{B}\right|^{p} d \gamma(x)\right]^{1 / p}<\infty,
$$

and $\Psi_{\kappa}(B)$ is given by setting, for all $B \in \mathcal{B}_{1}$,

$$
\Psi_{\kappa}(B)= \begin{cases}\left|c_{B}\right|^{2}+\ln \frac{2}{r_{B}}, & \kappa=0 \\ {\left[\frac{m\left(c_{B}\right)}{r_{B}}\right]^{-n \kappa / p},} & \kappa \in[-p / n, 0) .\end{cases}
$$

Moreover, the operator norm of $g$, which is denoted by $\|g\|_{\mathcal{M}^{p, \kappa}(\gamma) \rightarrow \mathcal{M}^{p, \kappa}(\gamma)}$, is comparable to $\|g\|_{L^{\infty}(\gamma)}+\|g\|_{\mathcal{L}_{\Psi_{\kappa}}^{p, \kappa}(\gamma)}$.

The proof of Theorem 1.2 is given in Section 4 below, based on some technical lemmas presented in Sections 2 and 3.

REMARK 1.2 .

(i) For all $p \in[1, \infty)$ and $\kappa \in[-p / n, 0]$, by Theorem 1.2 , we easily see that $C_{c}^{\infty}\left(\mathbb{R}^{n}\right)$ functions are pointwise multipliers on $\mathcal{L}^{p, \kappa}(\gamma)$.

(ii) The following fact is obvious: if $g \in L^{\infty}(\gamma)$ satisfies that there exists a positive constant $C$ such that, for all balls $B \in \mathcal{B}_{1}$ and almost all $x, y \in B$,

$$
|g(x)-g(y)| \leq \frac{C}{\left|c_{B}\right|^{2}+\ln \frac{2}{r_{B}}},
$$

then $g$ is a pointwise multiplier on $\operatorname{BMO}(\gamma)$.

(iii) For $p \in[1, \infty)$ and $\kappa \in[-p / n, 0)$, the space $\mathcal{L}_{\Psi_{\kappa}}^{p, \kappa}(\gamma)$ is indeed a "Lipschitz" space. For any $\alpha \in(0, \infty)$, the space $\dot{\Lambda}_{\alpha}(\gamma)$ is defined to be the collection of all functions $g$ such that

$$
\|g\|_{\dot{\Lambda}_{\alpha}(\gamma)}:=\sup _{|x-y| \leq m(x), x \neq y} \frac{|g(x)-g(y)|}{(1+|x|+|y|)^{\alpha}|x-y|^{\alpha}}<\infty .
$$

It is proved in Proposition 2.4 below that $\mathcal{L}_{\Psi_{\kappa}}^{p, \kappa}(\gamma)=\dot{\Lambda}_{-n \kappa / p}(\gamma)$ with equivalent seminorms. 
From Theorem 1.2 and the fact that the dual space of $H^{1}(\gamma)$ is $\operatorname{BMO}(\gamma)$, we immediately deduce the following conclusion. The details are omitted.

COROLlary 1.1. The following three statements are equivalent:

(i) $g$ is a pointwise multiplier on the space $\operatorname{BMO}(\gamma)$;

(ii) $g$ is a pointwise multiplier on the space $H^{1}(\gamma)$;

(iii) $g \in L^{\infty}(\gamma) \cap \mathrm{BMO}^{\log }(\gamma)$, where

$$
\|g\|_{\mathrm{BMO}^{\log (\gamma)}}:=\sup _{B \in \mathcal{B}_{1}} \frac{\left|c_{B}\right|^{2}+\ln \frac{2}{r_{B}}}{\gamma(B)} \int_{B}\left|g(x)-g_{B}\right| d \gamma(x)<\infty .
$$

Moreover, the operator norm of $g$ is comparable to $\|g\|_{L^{\infty}(\gamma)}+\|g\|_{\mathrm{BMO}^{\log (\gamma)}}$. In particular, functions in $C_{c}^{\infty}\left(\mathbb{R}^{n}\right)$ are pointwise multipliers on $\operatorname{BMO}(\gamma)$ and $H^{1}(\gamma)$.

By Corollary 1.1, we see that, if $f \in H^{1}(\gamma)$ and $g \in C_{c}^{\infty}\left(\mathbb{R}^{n}\right)$, then $f g \in H^{1}(\gamma)$. This is known to be true for the classical local Hardy space $h^{1}\left(\mathbb{R}^{n}\right)$ but false for the classical Hardy space $H^{1}\left(\mathbb{R}^{n}\right)$. In this sense, $H^{1}(\gamma)$ is local, and hence Corollary 1.1 has independent interest.

This article is organized as follows. In Section 2, we present some properties of the Campanato space, including its relations with the Lipschitz space, and also show that the space $\mathcal{L}_{\Psi_{\kappa}}^{p, \kappa}(\gamma)$ is indeed some "Lipschitz" space when $\kappa \in[-p / n, 0)$. Some auxiliary lemmas are established in Section 3. Theorems 1.1 and 1.2 are proved in Section 4. In Section 5, we give some examples of pointwise multipliers of the space $\operatorname{BMO}(\gamma)$. Particularly, in Example 5.4 below, applying Theorem 1.2, we find a nonnegative function which lies in $\operatorname{BMO}(\gamma)$ but not in $\operatorname{BLO}(\gamma)$. This further implies that $\{f \in \operatorname{BLO}(\gamma): f \geq 0\}$ is a proper subset of $\operatorname{BMO}(\gamma)$. Recall that it was proved in [19, Theorem 4.1] that the maximal singular integral $T^{*}$ is bounded from $L^{\infty}(\gamma)$ to $\{f \in \operatorname{BLO}(\gamma): f \geq 0\}$. We further know, from Example 5.4, that this result indeed improves the boundedness of $T^{*}$ from $L^{\infty}(\gamma)$ to $\operatorname{BMO}(\gamma)$.

We point out that this paper was inspired by the work of Nakai and Yabuta in [30] and [31]. However, due to the nondoubling property of the Gauss measure, we need several key properties of the Gauss measure developed in [23, Proposition 2.1, Lemma 5.1] and [18, Section 3.2], for example, the locally doubling property of admissible balls, and the geometric properties of maximal balls and their mothers. In particular, we need the following property. Given any two admissible balls being far away from each other, 
there exists a sequence of maximal balls connecting them, and the measure of each ball in this sequence has an estimate from below (see [18, Section 3.2] or Lemma 3.1 below).

Finally, we set some conventions. Let $\mathbb{N}:=\{1,2, \ldots\}$. Denote by $C$ a positive constant independent of the main parameters involved, which may vary at different occurrences. We use $f \lesssim h$ or $h \gtrsim f$ to denote $f \leq C h$ or $h \geq C f$, respectively. If $f \lesssim h \lesssim f$, we write $f \sim h$.

\section{§2. Properties of Campanato spaces}

For any cube $Q \subset \mathbb{R}^{n}$ and $p \in[1, \infty]$, denote by $L_{0}^{p}(Q ; \gamma)$ the collection of all $\phi \in L^{p}(\gamma)$ such that $\operatorname{supp} \phi \subset Q$ and $\int_{Q} \phi(x) d \gamma(x)=0$. The following proposition is proved in the spirit of [23, Proposition 2.4].

Proposition 2.1. Let $0<b<a<\infty$, let $p \in[1, \infty)$, and let $\kappa \in(-\infty, 1]$. Then

$$
\|f\|_{\mathcal{C}_{\mathcal{B}_{a}}^{p, \kappa}(\gamma)} \sim\|f\|_{\mathcal{C}_{\mathcal{Q} a}^{p, \kappa}(\gamma)} \sim\|f\|_{\mathcal{C}_{\mathcal{Q}_{b}}^{p, \kappa}(\gamma)} \sim\|f\|_{\mathcal{C}_{\mathcal{B}_{b}}^{p, \kappa}(\gamma)}
$$

with implicit positive constants depending only on $n, a, b, p$, and $\kappa$. Consequently, the spaces $\mathcal{L}_{\mathcal{B}_{a}}^{p, \kappa}(\gamma)=\mathcal{L}_{\mathcal{Q}_{a}}^{p, \kappa}(\gamma)=\mathcal{L}_{\mathcal{Q}_{b}}^{p, \kappa}(\gamma)=\mathcal{L}_{\mathcal{B}_{b}}^{p, \kappa}(\gamma)$ with equivalent norms.

Proof. By a reexamination of the proof of [23, Lemma 2.3], we see that there exist a positive constant $C$ and a nonnegative integer $N$, depending only on $n, p, a$, and $b$, such that for any cube $Q$ in $\mathcal{Q}_{a}$ and function $\phi \in$ $L_{0}^{p^{\prime}}(Q ; \gamma)$, there exist at most $N$ subcubes $\left\{Q_{1}, \ldots, Q_{N}\right\}$ in the admissible class $\mathcal{Q}_{b}$ and $N$ functions $\left\{\phi_{1}, \ldots, \phi_{N}\right\}$ in $L_{0}^{p^{\prime}}(Q ; \gamma)$, with supports contained in $\left\{Q_{1}, \ldots, Q_{N}\right\}$, respectively, such that

$\phi=\sum_{j=1}^{N} \phi_{j}, \quad\left\|\phi_{j}\right\|_{L^{p}(\gamma)} \leq C\|\phi\|_{L^{p}(\gamma)}, \quad$ and $\quad \frac{1}{C} \gamma(Q) \leq \gamma\left(Q_{j}\right) \leq \gamma(Q)$

The rest of the proof is parallel to that of [23, Proposition 2.4], and the details are left to the reader.

The following estimate is needed in the proofs of Proposition 2.3 and Lemma 3.2 below. 
Proposition 2.2. Let $p \in[1, \infty)$, and let $\kappa \in(-\infty, 0]$. If $f \in \mathcal{C}^{p, \kappa}(\gamma)$, then for all $B, B^{\prime} \in \mathcal{B}_{1}$ with $c_{B}=c_{B^{\prime}}$ and $r_{B}<r_{B^{\prime}}$, it holds that

$$
\left|f_{B}-f_{B^{\prime}}\right| \leq \begin{cases}C\left[1+\ln \frac{r_{B^{\prime}}}{r_{B}}\right]\|f\|_{\mathcal{C}^{p, \kappa}(\gamma)}, & \kappa=0, \\ C\left[\frac{r_{B}}{r_{B^{\prime}}}\right]^{n \kappa / p} \frac{\|f\|_{\mathcal{C}^{p, \kappa}(\gamma)}}{[\gamma(B)]^{\kappa / p}}, & \kappa \in(-\infty, 0),\end{cases}
$$

where $C$ is a positive constant depending only on $p, \kappa$, and $n$.

Proof. We show (2.1) by considering the following two cases: $r_{B} \geq r_{B^{\prime}} / 2$ and $r_{B}<r_{B^{\prime}} / 2$.

Case 1: $r_{B} \geq r_{B^{\prime}} / 2$. In this case, we have $B \subset B^{\prime} \subset 2 B$, and hence $\gamma(B) \sim$ $\gamma\left(B^{\prime}\right)$ by (1.4). From this and Hölder's inequality, we deduce that

$$
\left|f_{B}-f_{B^{\prime}}\right| \leq \frac{1}{\gamma(B)} \int_{B}\left|f(x)-f_{B^{\prime}}\right| d \gamma(x) \lesssim \frac{\|f\|_{\mathcal{C}^{p, \kappa}(\gamma)}}{[\gamma(B)]^{\kappa / p}}
$$

which implies that (2.1) holds.

Case 2: $r_{B}<r_{B^{\prime}} / 2$. In this case, we choose $j_{0} \geq 1$ such that $2^{j_{0}} r_{B} \leq r_{B^{\prime}}<$ $2^{j_{0}+1} r_{B}$. For $j \in\left\{1, \ldots, j_{0}\right\}$, let $B_{j}:=B\left(c_{B}, 2^{j} r_{B}\right)$. Write

$$
\left|f_{B}-f_{B^{\prime}}\right| \leq \sum_{j=0}^{j_{0}-1}\left|f_{B_{j}}-f_{B_{j+1}}\right|+\left|f_{B_{j_{0}}}-f_{B^{\prime}}\right|
$$

For all $j \in\left\{1, \ldots, j_{0}-1\right\}$, by $r_{B_{j}}=r_{B_{j+1}} / 2$ and $(2.2)$, we conclude that

$$
\left|f_{B_{j}}-f_{B_{j+1}}\right| \lesssim \frac{\|f\|_{\mathcal{C}^{p, \kappa}(\gamma)}}{\left[\gamma\left(B_{j}\right)\right]^{\kappa / p}}
$$

Similarly, $\left|f_{B_{j_{0}}}-f_{B^{\prime}}\right|$ has the same upper bound as in (2.3). Thus, by summing the inequalities (2.3), we know that

$$
\left|f_{B}-f_{B^{\prime}}\right| \lesssim \sum_{j=0}^{j_{0}} \frac{\|f\|_{\mathcal{C}^{p, \kappa}(\gamma)}}{\left[\gamma\left(B_{j}\right)\right]^{\kappa / p}} \lesssim \frac{\|f\|_{\mathcal{C}^{p, \kappa}(\gamma)}}{[\gamma(B)]^{\kappa / p}} \sum_{j=0}^{j_{0}} \frac{1}{2^{j n \kappa / p}}
$$

where we used the fact that $\gamma\left(B_{j}\right) \sim 2^{j n} \gamma(B)$. Then, applying $2^{j 0} \sim r_{B^{\prime}} / r_{B}$, we see that $(2.1)$ holds for the case $r_{B}<r_{B^{\prime}} / 2$. Hence, we conclude the proof of Proposition 2.2.

Using Propositions 2.1 and 2.2, we now establish the coincidence between $\mathcal{C}^{p, \kappa}(\gamma)$ and $\operatorname{Lip}_{-\kappa / p}(\gamma)$ for $p \in[1, \infty)$ and $\kappa \in[-p / n, 0)$. 
Proposition 2.3. Let $p \in[1, \infty)$. Then, the following hold:

(i) when $\kappa \in[-p / n, 0), \mathcal{C}^{p, \kappa}(\gamma)=\operatorname{Lip}_{-\kappa / p}(\gamma)$ with equivalent seminorms;

(ii) when $\kappa \in(-\infty,-p / n), \mathcal{C}^{p, \kappa}(\gamma)$ consists only of functions which are constant almost everywhere.

Proof. For $\kappa \in[-p / n, 0)$, it is easy to see that $\operatorname{Lip}_{-\kappa / p}(\gamma) \subset \mathcal{C}^{p, \kappa}(\gamma)$ by their definitions. Also, when $\kappa \in(-\infty,-p / n)$, it is obvious that almost everywhere constant functions belong to $\mathcal{C}^{p, \kappa}(\gamma)$.

Let $f \in \mathcal{C}^{p, \kappa}(\gamma)$ with $\kappa \in(-\infty, 0)$. For any $B \in \mathcal{B}_{1}$ and almost every $x, y \in B$, by the differential theorem of integrals, we see that

$$
\begin{aligned}
|f(x)-f(y)|= & \lim _{j \rightarrow \infty}\left|f_{B\left(x, 2^{-j} r_{B}\right)}-f_{B\left(y, 2^{-j} r_{B}\right)}\right| \\
\leq & \lim _{j \rightarrow \infty}\left[\left|f_{B\left(x, 2^{-j} r_{B}\right)}-f_{B\left(x, r_{B}\right)}\right|\right. \\
& \left.+\left|f_{B\left(x, r_{B}\right)}-f_{B\left(y, r_{B}\right)}\right|+\left|f_{B\left(y, r_{B}\right)}-f_{B\left(y, 2^{-j} r_{B}\right)}\right|\right] .
\end{aligned}
$$

From Proposition 2.2 and $\gamma\left(B\left(x, 2^{-j} r_{B}\right)\right) \sim 2^{-j n} \gamma(B)$, we deduce that

$$
\left|f_{B\left(x, 2^{-j} r_{B}\right)}-f_{B\left(x, r_{B}\right)}\right| \lesssim \frac{\|f\|_{\mathcal{C}^{p, \kappa}(\gamma)}}{\left[\gamma\left(B\left(x, 2^{-j} r_{B}\right)\right)\right]^{\kappa / p}} \frac{1}{2^{j n \kappa / p}} \lesssim \frac{\|f\|_{\mathcal{C}^{p, \kappa}(\gamma)}}{[\gamma(B)]^{\kappa / p}}
$$

Likewise, for almost every $y \in B$, we have

$$
\left|f_{B\left(y, 2^{-j} r_{B}\right)}-f_{B\left(y, r_{B}\right)}\right| \lesssim \frac{\|f\|_{\mathcal{C}^{p, \kappa}(\gamma)}}{[\gamma(B)]^{\kappa / p}}
$$

For all $x, y \in B$, we observe that $B\left(x, r_{B}\right) \subset 2 B, B\left(y, r_{B}\right) \subset 2 B$, and hence, by $(1.2)$ and (1.3), $\gamma(B) \sim \gamma\left(B\left(x, r_{B}\right)\right) \sim \gamma\left(B\left(y, r_{B}\right)\right)$. From these and Proposition 2.1, it follows that

$$
\begin{aligned}
\left|f_{B\left(x, r_{B}\right)}-f_{B\left(y, r_{B}\right)}\right| & \leq\left|f_{B\left(x, r_{B}\right)}-f_{2 B}\right|+\left|f_{2 B}-f_{B\left(y, r_{B}\right)}\right| \\
& \lesssim \frac{\|f\|_{\mathcal{C}^{p, \kappa}(\gamma)}}{[\gamma(B)]^{\kappa / p}} .
\end{aligned}
$$

Inserting (2.5), (2.6), and (2.7) into (2.4), and using (1.3), we see that, for almost every $x, y \in B \in \mathcal{B}_{a}$,

$$
|f(x)-f(y)| \lesssim \frac{\|f\|_{\mathcal{C}^{p, \kappa}(\gamma)}}{[\gamma(B)]^{\kappa / p}} \sim\|f\|_{\mathcal{C}^{p, \kappa}(\gamma)} e^{\kappa\left|c_{B}\right|^{2} / p} r_{B}^{-n \kappa / p}
$$


which implies that $f \in \operatorname{Lip}_{-\kappa / p}(\gamma)$ when $\kappa \in[-p / n, 0)$. Meanwhile, when $\kappa \in(-\infty,-p / n)$, from (2.8) one deduces that the derivative of $f$ is 0 for almost every $x \in B$, and hence $f$ is a constant function almost everywhere. This finishes the proof of Proposition 2.3.

Proposition 2.4. Let $p \in[1, \infty)$, and let $\kappa \in[-p / n, 0)$. Then, $\mathcal{L}_{\Psi_{\kappa}}^{p, \kappa}(\gamma)=$ $\dot{\Lambda}_{-n \kappa / p}(\gamma)$ with equivalent seminorms, where $\Psi_{\kappa}$ is as in (1.6).

Proof. Using the fact that $m(x)^{-1} \sim 1+|x| \sim 1+|y| \sim m(y)^{-1}$ when $x, y \in \mathbb{R}^{n}$ and $|x-y| \leq m(x)$, we conclude that $g \in \dot{\Lambda}_{-n \kappa / p}(\gamma)$ if and only if, for all $x, y \in B \in \mathcal{B}_{1}$,

$$
|g(x)-g(y)| \lesssim\left[\frac{r_{B}}{m\left(c_{B}\right)}\right]^{-n \kappa / p} .
$$

It is obvious that any function $g$ that satisfies (2.9) implies that $g \in \mathcal{L}_{\Psi_{\kappa}}^{p, \kappa}(\gamma)$. Thus, $\dot{\Lambda}_{-n \kappa / p}(\gamma) \subset \mathcal{L}_{\Psi_{\kappa}}^{p, \kappa}(\gamma)$. The converse follows from an argument similar to the second part of the proof of Proposition 2.3; the details are left to the reader. This finishes the proof.

\section{$\S 3$. Some auxiliary lemmas}

In this section, we establish some technical lemmas to be used in the proof of Theorem 1.2. We begin with recalling some notions from [23]. A ball $B \in \mathcal{B}_{1}$ is said to be maximal if $r_{B}=m\left(c_{B}\right)$. For any maximal ball $B \in \mathcal{B}_{1}$ such that $0 \notin B$, denote by $M(B)$ the maximal ball in $\mathcal{B}_{1}$ centered at a point on the segment $\left[0, c_{B}\right]=\left\{t c_{B}: t \in[0,1]\right\}$ such that the boundary of $M(B)$ contains $c_{B}$. The ball $M(B)$ is called the mother of $B$. Then, for any maximal ball $B \in \mathcal{B}_{1}$ that satisfies that $0 \notin B$, we have

$$
\frac{1}{3} M(B) \subset B \subset 3 M(B) \quad \text { and } \quad \gamma(M(B)) \sim \gamma(B)
$$

with implicit positive constants depending only on $n$ (see [18]).

For notational convenience, we let $M^{0}(B):=B$ for any maximal ball $B \in \mathcal{B}_{1}$. If $0 \notin M(B)$, then we consider the mother of $M(B)$ and denote it by $M^{2}(B)$. Thus, there exists a chain of maximal balls, $B, M(B), M^{2}(B)$, $\ldots, M^{k}(B)$, with the property that $M^{j}(B)$ is the mother of $M^{j-1}(B)$ for $j \in$ $\{1, \ldots, k\}$, and $M^{k}(B) \ni 0$. The following lemma concerning the properties of such chains of maximal balls was proved in [18, Section 3.2]. 
LEMMA 3.1. Let $B$ be a maximal ball in $\mathcal{B}_{1}$, and let $\left\{a_{i}\right\}_{i \in \mathbb{N}}$ be the sequence recursively defined by $a_{0}:=1$ and $a_{i+1}:=a_{i}+1 / a_{i}$ for $i \in \mathbb{N} \cup\{0\}$. Then, the following hold:

(i) for all $j \in \mathbb{N}, \sqrt{2 j} \leq a_{j} \leq \sqrt{3 j}$;

(ii) if $a_{j} \leq\left|c_{B}\right|<a_{j+1}$ for some $j \in \mathbb{N}$, then either $\left|c_{M(B)}\right| \leq 2$ or $a_{j-1} \leq$ $\left|c_{M(B)}\right|<a_{j}$

(iii) if $a_{j} \leq\left|c_{B}\right|<a_{j+1}$ for some $j \in \mathbb{N}$, then $\gamma(B) \sim e^{-a_{j}^{2}} j^{-n / 2}$;

(iv) if $\left|c_{B}\right|>2$ and $\left\{M^{k}(B)\right\}_{k=0}^{k_{0}}$ is a chain of maximal balls in $\mathcal{B}_{1}$, with the property that $M^{k}(B)$ is the mother of $M^{k-1}(B)$ for $k \in\left\{1, \ldots, k_{0}\right\}$, where $k_{0}$ is the smallest integer such that $\left|c_{M^{k_{0}(B)}}\right| \leq 2$, then for all $k \in\left\{1, \ldots, k_{0}\right\}, \gamma(B) \lesssim e^{-2 k} \gamma\left(M^{k}(B)\right)$.

Here the implicit positive constants in (i)-(iv) depend only on $n$.

From Lemma 3.1, we deduce the following estimate.

Lemma 3.2. Let $p \in[1, \infty)$, and let $\kappa \in[-p / n, 0]$. Then there exists a positive constant $C$ such that, for all $f \in \mathcal{L}^{p, \kappa}(\gamma)$ and $B \in \mathcal{B}_{1}$,

$$
\left|f_{B}\right| \leq C\left[\|f\|_{L^{1}(\gamma)}+\Psi_{\kappa}(B)[\gamma(B)]^{-\kappa / p}\|f\|_{\mathcal{C}^{p, \kappa}(\gamma)}\right]
$$

Proof. To prove this lemma, we consider the following two cases: $\left|c_{B}\right| \leq 2$ and $\left|c_{B}\right|>2$.

Case 1: $\left|c_{B}\right| \leq 2$. In this case, we have $m\left(c_{B}\right) \geq 1 / 2$ and $B\left(c_{B}, 1\right) \in \mathcal{B}_{2}$. Combining this with (2.1) and (1.6) implies that

$$
\left|f_{B}-f_{B\left(c_{B}, 1\right)}\right| \lesssim \begin{cases}{\left[1+\ln \frac{1}{r_{B}}\right]\|f\|_{\mathcal{C}^{p, \kappa}(\gamma)},} & \kappa=0 \\ {\left[r_{B}\right]^{n \kappa / p} \frac{\|f\|_{\mathcal{C}^{p, \kappa}(\gamma)}}{[\gamma(B)]^{\kappa / p}},} & \kappa \in(-p / n, 0)\end{cases}
$$

Noticing that $\gamma\left(B\left(c_{B}, 1\right)\right) \sim 1$, we have

$$
\left|f_{B\left(c_{B}, 1\right)}\right| \leq \frac{1}{\gamma\left(B\left(c_{B}, 1\right)\right)} \int_{B\left(c_{B}, 1\right)}|f(x)| d \gamma(x) \lesssim\|f\|_{L^{1}(\gamma)} .
$$

Adding these two estimates and using (1.6) and (1.1), we obtain (3.2).

Case 2: $\left|c_{B}\right|>2$. In this case, $m\left(c_{B}\right)=1 /\left|c_{B}\right|$ and $B_{0}:=B\left(c_{B}, 1 /\left|c_{B}\right|\right)$ is a maximal ball in $\mathcal{B}_{1}$. Since the sequence $\left\{a_{j}\right\}_{j=0}^{\infty}$ in Lemma 3.1 is increasing to infinity and $a_{1}=2$, there exists some $j_{0} \in \mathbb{N}$ such that $a_{j_{0}} \leq\left|c_{B}\right|<$ $a_{j_{0}+1}$. Notice that $j_{0} \sim\left|c_{B}\right|^{2}$ by Lemma 3.1(i). For $j \in\left\{0, \ldots, j_{0}\right\}$, let $B_{j}:=$ 
$M^{j}\left(B_{0}\right)$. Then, $B_{j+1}$ is the mother of $B_{j}$. Write

$$
\left|f_{B}\right| \leq\left|f_{B}-f_{B_{0}}\right|+\sum_{j=0}^{j_{0}-1}\left|f_{B_{j}}-f_{B_{j+1}}\right|+\left|f_{B_{j_{0}}}\right| .
$$

If $r_{B}=m\left(c_{B}\right)=1 /\left|c_{B}\right|$, then $B=B_{0}$ and $\left|f_{B}-f_{B_{0}}\right|=0$; otherwise, Proposition 2.2 implies that

$$
\left|f_{B}-f_{B_{0}}\right| \lesssim \begin{cases}{\left[1+\ln \frac{m\left(c_{B}\right)}{r_{B}}\right]\|f\|_{\mathcal{C}^{p, \kappa}(\gamma)},} & \kappa=0, \\ {\left[\frac{r_{B}}{m\left(c_{B}\right)}\right]^{n \kappa / p} \frac{\|f\|_{\mathcal{C}^{p, \kappa}(\gamma)}}{[\gamma(B)]^{\kappa / p}},} & \kappa \in(-\infty, 0) .\end{cases}
$$

According to Lemma 3.1(ii), for all $j \in\left\{0, \ldots, j_{0}\right\}$, we have

$$
a_{j_{0}-j} \leq\left|c_{B_{j}}\right|<a_{j_{0}+1-j}
$$

Specifically, it holds that $\left|c_{B_{j_{0}}}\right|<a_{1}=2$ and $\gamma\left(B_{j_{0}}\right) \sim 1$, which implies that

$$
\left|f_{B_{j_{0}}}\right| \lesssim\|f\|_{L^{1}(\gamma)}
$$

For $j \in\left\{0, \ldots, j_{0}-1\right\}$, since $B_{j+1}$ is the mother of the ball $B_{j}$ and they are both maximal balls in $\mathcal{B}_{1}$, we apply (3.1) to conclude that $B_{j} \subset 3 B_{j+1}$, $B_{j+1} \subset 3 B_{j}$, and $\gamma\left(B_{j}\right) \sim \gamma\left(B_{j+1}\right)$. Then, by Hölder's inequality, (1.4), and Proposition 2.1, we further see that

$$
\begin{aligned}
\sum_{j=0}^{j_{0}-1}\left|f_{B_{j}}-f_{B_{j+1}}\right| & \leq \sum_{j=0}^{j_{0}-1}\left[\left|f_{B_{j}}-f_{3 B_{j+1}}\right|+\left|f_{3 B_{j+1}}-f_{B_{j+1}}\right|\right] \\
& \lesssim\|f\|_{\mathcal{C}^{p, \kappa}(\gamma)} \sum_{j=0}^{j_{0}-1} \frac{1}{\left[\gamma\left(B_{j}\right)\right]^{\kappa / p}} .
\end{aligned}
$$

In particular, when $\kappa=0$, we have

$$
\sum_{j=0}^{j_{0}-1}\left|f_{B_{j}}-f_{B_{j+1}}\right| \lesssim\|f\|_{\mathcal{C}^{p, \kappa}(\gamma)} j_{0} \lesssim\|f\|_{\mathcal{C}^{p, \kappa}(\gamma)}\left|c_{B}\right|^{2}
$$

When $\kappa<0$, from (3.5) and Lemma 3.1(i), (iii), we infer that 


$$
\begin{aligned}
\sum_{j=0}^{j_{0}-1}\left|f_{B_{j}}-f_{B_{j+1}}\right| & \lesssim\|f\|_{\mathcal{C}^{p, \kappa}(\gamma)} \sum_{j=0}^{j_{0}-1}\left[e^{-\left(a_{j_{0}-j}\right)^{2}}\left(j_{0}-j\right)^{n / 2}\right]^{|\kappa| / p} \\
& \lesssim\|f\|_{\mathcal{C}^{p, \kappa}(\gamma)} \sum_{j=1}^{j_{0}}\left[e^{-2 j} j^{n / 2}\right]^{|\kappa| / p} \\
& \lesssim\left[\frac{r_{B}}{m\left(c_{B}\right)}\right]^{n \kappa / p} \frac{\|f\|_{\mathcal{C}^{p, \kappa}(\gamma)}}{[\gamma(B)]^{\kappa / p}}
\end{aligned}
$$

Inserting the estimates (3.4), (3.6), (3.7), and (3.8) into (3.3), we see that (3.2) holds also when $\left|c_{B}\right|>2$. This finishes the proof of Lemma 3.2.

In the next two lemmas, we construct two examples of functions in the space $\mathcal{L}^{p, \kappa}(\gamma)$ with $\kappa \in[-p / n, 1]$ and $\kappa \neq p$. These two examples are used to prove the necessity of Theorem 1.2 .

Lemma 3.3. Let $p \in[1, \infty)$, let $\kappa \in[-p / n, 1]$, and let $\kappa \neq p$. Given any ball $B \in \mathcal{B}_{1}$, define

$$
f(x):=\max \left\{0, \int_{\left|x-c_{B}\right|}^{4 m\left(c_{B}\right)} \frac{1}{\left[e^{\left.-\left|c_{B}\right|^{2} t^{n}\right]^{\kappa / p}}\right.} \frac{d t}{t}\right\}, \quad x \in \mathbb{R}^{n} .
$$

Then, there exists a positive constant $C$, independent of the ball $B$, such that

(i) if $\kappa \in[-p / n, 0)$, then $\|f\|_{\mathcal{L}^{p, \kappa}(\gamma)} \leq C$ and $f(x) \geq C \Psi_{\kappa}(B) /[\gamma(B)]^{\kappa / p}$ for all $x \in B$;

(ii) if $\kappa=0$, then $\|f\|_{\mathrm{BMO}(\gamma)} \leq C$ and $f(x) \geq C \ln \left(2 / r_{B}\right)$ for all $x \in B$;

(iii) if $\kappa \in(0,1] \cap(0, p)$, then $\|f\|_{\mathcal{L}^{p, \kappa}(\gamma)} \leq C$ and $f(x) \geq C /[\gamma(B)]^{\kappa / p}$ for all $x \in B$.

Proof. Recall that $\Psi_{\kappa}(B)=\left(m\left(c_{B}\right) / r_{B}\right)^{-n \kappa / p}$ when $\kappa \in[-n / p, 0)$. For all $x \in B$, since $\left|x-c_{B}\right|<r_{B}$, it follows that

$$
\begin{aligned}
f(x) & \geq \int_{\left|x-c_{B}\right|}^{4 m\left(c_{B}\right)} \frac{1}{\left[e^{\left.-\left|c_{B}\right|^{2} t^{n}\right]^{\kappa / p}} \frac{d t}{t} \sim \frac{1}{[\gamma(B)]^{\kappa / p}} \int_{\frac{\left|x-c_{B}\right|}{r_{B}}}^{\frac{4 m\left(c_{B}\right)}{r_{B}}} s^{-n \kappa / p-1} d s\right.} \\
& \gtrsim \begin{cases}\left(\frac{m\left(c_{B}\right)}{r_{B}}\right)^{-n \kappa / p} \frac{1}{[\gamma(B)]^{\kappa / p}}, & \kappa<0, \\
\ln \frac{2}{r_{B}}, & \kappa=0, \\
\frac{1}{[\gamma(B)]^{\kappa / p}}, & \kappa>0,\end{cases}
\end{aligned}
$$

which implies the second conclusions of (i), (ii), and (iii). 
To show the remaining conclusions of (i), (ii), and (iii), we first show that $\|f\|_{L^{1}(\gamma)} \lesssim 1$. Observing that $\operatorname{supp} f \subset B\left(c_{B}, 4 m\left(c_{B}\right)\right)$ and using Fubini's theorem, we find that

$$
\begin{aligned}
\|f\|_{L^{1}(\gamma)} & =\int_{B\left(c_{B}, 4 m\left(c_{B}\right)\right)}\left[\int_{\left|x-c_{B}\right|}^{4 m\left(c_{B}\right)} \frac{1}{\left[e^{-\left|c_{B}\right|^{2}} t^{n}\right]^{\kappa / p}} \frac{d t}{t}\right] d \gamma(x) \\
& =\int_{0}^{4 m\left(c_{B}\right)} \frac{\gamma\left(B\left(c_{B}, t\right)\right)}{\left[e^{-\left|c_{B}\right|^{2}} t^{n}\right]^{\kappa / p}} \frac{d t}{t} .
\end{aligned}
$$

For all $t \in\left[0,4 m\left(c_{B}\right)\right]$, we see that $B\left(c_{B}, t\right) \in \mathcal{B}_{4}$, and hence $\gamma\left(B\left(c_{B}, t\right)\right) \sim$ $e^{-\left|c_{B}\right|^{2} t^{n}}$ by (1.3). Also, notice that $4 m\left(c_{B}\right) \leq 4$. We continue the above estimate and conclude that

$$
\|f\|_{L^{1}(\gamma)} \sim \int_{0}^{4 m\left(c_{B}\right)} e^{-(1-\kappa / p)\left|c_{B}\right|^{2}} t^{n(1-\kappa / p)-1} d t \lesssim \int_{0}^{4} t^{n(1-\kappa / p)-1} d t \lesssim 1,
$$

where the last step is due to the fact that $n(1-\kappa / p)>0$ for all $\kappa \in[-p / n, 1]$ and $\kappa \neq p$. This proves that $f \in L^{1}(\gamma)$. Thus, to prove the first conclusions of (i), (ii), and (iii), we need only to show that $\|f\|_{\mathcal{C}^{p, \kappa}(\gamma)} \lesssim 1$.

The proof of the remaining part of (i) can be quite simple by using Proposition 2.3. Indeed, when $\kappa \in[-p / n, 0)$, applying the trivial inequality

$$
|\max \{0, a\}-\max \{0, b\}| \leq|a-b| \text { for all } a, b \in \mathbb{R},
$$

we conclude that, when $\kappa \in[-p / n, 0)$, for any ball $B \in \mathcal{B}_{1}$ and $x, y \in B$, it holds that

$$
\begin{aligned}
|f(x)-f(y)| & \leq\left|\int_{\left|y-c_{B}\right|}^{\left|x-c_{B}\right|} \frac{1}{\left[e^{\left.-\left|c_{B}\right|^{2} t^{n}\right]^{\kappa / p}}\right.} \frac{d t}{t}\right| \sim \frac{1}{[\gamma(B)]^{\kappa / p}}\left|\int_{\frac{\left|y-c_{B}\right|}{r_{B}}}^{\frac{\left|x-c_{B}\right|}{r_{B}}} s^{-n \kappa / p} \frac{d s}{s}\right| \\
& \lesssim \frac{1}{[\gamma(B)]^{\kappa / p}},
\end{aligned}
$$

which implies that $\|f\|_{\operatorname{Lip}_{-\kappa / p}(\gamma)} \lesssim 1$. Hence, $\|f\|_{\mathcal{C}^{p, \kappa}(\gamma)} \lesssim 1$ by Proposition 2.3. This completes the proof of (i).

We treat (ii) and (iii) in a unified way. When $\kappa \in[0,1]$ and $\kappa \neq p$, to show that $\|f\|_{\mathcal{C}^{p, \kappa}(\gamma)} \lesssim 1$, it suffices to show that, for any $B^{\prime} \in \mathcal{B}_{1}$, there exists some $A_{B^{\prime}} \in \mathbb{C}$ such that

$$
\left\{\frac{1}{\left[\gamma\left(B^{\prime}\right)\right]^{1-\kappa}} \int_{B^{\prime}}\left|f(x)-A_{B^{\prime}}\right|^{p} d \gamma(x)\right\}^{1 / p} \leq C,
$$

where $C$ is a positive constant independent of $B$ and $B^{\prime}$. To this end, we consider the following two cases: $\left|c_{B}-c_{B^{\prime}}\right| \leq 2 r_{B^{\prime}}$ and $\left|c_{B}-c_{B^{\prime}}\right|>2 r_{B^{\prime}}$. 
Case 1: $\left|c_{B}-c_{B^{\prime}}\right| \leq 2 r_{B^{\prime}}$. In this case, choose

$$
A_{B^{\prime}}:=\max \left\{0, \int_{r_{B^{\prime}}}^{4 m\left(c_{B}\right)} \frac{1}{\left[e^{-\left|c_{B}\right|^{2}} t^{n}\right]^{\kappa / p}} \frac{d t}{t}\right\} \text {. }
$$

For all $x \in B^{\prime}$, we have $\left|x-c_{B}\right| \leq\left|x-c_{B^{\prime}}\right|+\left|c_{B^{\prime}}-c_{B}\right|<3 r_{B^{\prime}}$. Since here $\left|c_{B}-c_{B^{\prime}}\right| \leq 2 r_{B^{\prime}}$ and $B^{\prime} \in \mathcal{B}_{1}$, by (1.2) and (1.3), we will then see that we have $e^{-\left|c_{B}\right|^{2}}\left(r_{B^{\prime}}\right)^{n} \sim e^{-\left|c_{B^{\prime}}\right|^{2}}\left(r_{B^{\prime}}\right)^{n} \sim \gamma\left(B^{\prime}\right)$. Combining these with (3.9), we conclude that, for all $x \in B^{\prime}$,

$$
\begin{aligned}
\left|f(x)-A_{B^{\prime}}\right| & \leq\left|\int_{r_{B^{\prime}}}^{\left|x-c_{B}\right|} \frac{1}{\left[e^{\left.-\left|c_{B}\right|^{2} t^{n}\right]^{\kappa / p}}\right.} \frac{d t}{t}\right| \leq \int_{r_{B^{\prime}}}^{3 r_{B^{\prime}}} \frac{1}{\left[e^{\left.-\left|c_{B}\right|^{2} t^{n}\right]^{\kappa / p}}\right.} \frac{d t}{t} \\
& \lesssim \frac{1}{\left[\gamma\left(B^{\prime}\right)\right]^{\kappa / p}},
\end{aligned}
$$

which implies that (3.10) holds when $\left|c_{B}-c_{B^{\prime}}\right| \leq 2 r_{B^{\prime}}$.

Case 2: $\left|c_{B}-c_{B^{\prime}}\right|>2 r_{B^{\prime}}$. In this case, choose

$$
A_{B^{\prime}}:=\max \left\{0, \int_{\left|c_{B}-c_{B^{\prime}}\right|}^{4 m\left(c_{B}\right)} \frac{1}{\left[e^{\left.-\left|c_{B}\right|^{2} t^{n}\right]^{\kappa / p}}\right.} \frac{d t}{t}\right\} \text {. }
$$

For all $x \in B^{\prime}$, using (3.9) and $\left|c_{B}-c_{B^{\prime}}\right| / 2 \leq\left|x-c_{B}\right| \leq(3 / 2)\left|c_{B}-c_{B^{\prime}}\right|$, we know that

$$
\begin{aligned}
\left|f(x)-A_{B^{\prime}}\right| & \leq\left|\int_{\left|c_{B}-c_{B^{\prime}}\right|}^{\left|x-c_{B}\right|} \frac{1}{\left[e^{\left.-\left|c_{B}\right|^{2} t^{n}\right]^{\kappa / p}}\right.} \frac{d t}{t}\right| \\
& \lesssim \frac{r_{B^{\prime}}}{e^{-\left|c_{B}\right|^{2} \kappa / p}\left|c_{B}-c_{B^{\prime}}\right|^{n \kappa / p+1}} .
\end{aligned}
$$

If $x \in B^{\prime}$ and $\left|f(x)-A_{B^{\prime}}\right| \neq 0$, then either we have $\left|x-c_{B}\right|<4 m\left(c_{B}\right)$ or $\left|c_{B}-c_{B^{\prime}}\right|<4 m\left(c_{B}\right)$; whatever the case, we have $e^{-\left|c_{B}\right|^{2}} \sim e^{-|x|^{2}} \sim e^{-\left|c_{B^{\prime}}\right|^{2}}$ by (1.1) and (1.2). By this, (3.11), and the facts that $\gamma\left(B^{\prime}\right) \sim e^{-\left|c_{B^{\prime}}\right|^{2}} r_{B^{\prime}}^{n}$ and that $n \kappa / p+1>0$, we see that

the left-hand side of $(3.10) \lesssim\left\{\frac{1}{\left[\gamma\left(B^{\prime}\right)\right]^{-\kappa}} \frac{r_{B^{\prime}}^{p}}{e^{-\left|c_{B}\right|^{2} \kappa}\left|c_{B}-c_{B^{\prime}}\right|^{n \kappa+p}}\right\}^{1 / p}$

$$
\lesssim\left\{\frac{r_{B^{\prime}}}{\left|c_{B}-c_{B^{\prime}}\right|}\right\}^{n \kappa / p+1} \lesssim 1
$$

which completes the proofs of (ii) and (iii), as well the proof of Lemma 3.3.

LEMMA 3.4. Given any ball $B \in \mathcal{B}_{1}$, we are able to define $h(x):=|x|^{2}+\max \left\{0, \ln \frac{4 m\left(c_{B}\right)}{\left|x-c_{B}\right|}\right\}$ for all $x \in \mathbb{R}^{n}$. Then we have positive con- 
stant $C$, independent of the ball $B$, such that $\|h\|_{\mathrm{BMO}(\gamma)} \leq C$ and $h(x) \geq$ $C\left(\left|c_{B}\right|^{2}+\ln \left(2 / r_{B}\right)\right)$ for all $x \in B$.

Proof. For all $x \in \mathbb{R}^{n}$, let $h_{1}(x):=|x|^{2}$ and $h_{2}(x):=\max \left\{0, \ln \frac{4 m\left(c_{B}\right)}{\left|x-c_{B}\right|}\right\}$. Observe that $\left\|h_{2}\right\|_{\mathrm{BMO}(\gamma)} \lesssim 1$ by Lemma 3.3(ii). It is easy to see that $h_{1} \in$ $L^{1}(\gamma)$. For all $B^{\prime} \in \mathcal{B}_{1}$, by the fact that $r_{B^{\prime}} \leq m\left(c_{B^{\prime}}\right) \leq 1 /\left|c_{B^{\prime}}\right|$, we have $r_{B^{\prime}}\left|c_{B^{\prime}}\right| \leq 1$, and hence, for all $x, y \in B^{\prime}$,

$$
\left|h_{1}(x)-h_{1}(y)\right| \leq|x-y||x+y|<2 r_{B^{\prime}}\left(\left|x-c_{B^{\prime}}\right|+\left|y-c_{B^{\prime}}\right|+2\left|c_{B^{\prime}}\right|\right) \leq 8 .
$$

This further implies that $\left\|h_{1}\right\|_{\mathrm{BMO}(\gamma)} \lesssim 1$. Thus, $\|h\|_{\mathrm{BMO}(\gamma)} \lesssim 1$.

For all $B \in \mathcal{B}_{1}$ and $x \in B$, observing that $1+|x|^{2} \sim 1+\left|c_{B}\right|^{2}$, we apply Lemma 3.3(ii) to deduce that $h(x) \gtrsim\left|c_{B}\right|^{2}+\ln \left(2 / r_{B}\right)$. This concludes the proof of the lemma.

The proof for the following lemma is essentially given by Nakai and Yabuta in [31, Lemma 3.4] (see also [30, Lemma 3.3], [37, p. 582]). We omit the details.

Lemma 3.5. Suppose that $p \in[1, \infty)$ and that $\kappa \in(-\infty, 1]$. If $f \in \mathcal{C}^{p, \kappa}(\gamma)$ and $g \in L^{\infty}(\gamma)$, then $f g \in \mathcal{C}^{p, \kappa}(\gamma)$ if and only if

$$
F(f, g):=\sup _{B \in \mathcal{B}_{1}}\left|f_{B}\right|\left[\frac{1}{[\gamma(B)]^{1-\kappa}} \int_{B}\left|g(x)-g_{B}\right|^{p} d \gamma(x)\right]^{1 / p}<\infty .
$$

In this case, $\left|\|f g\|_{\mathcal{C}^{p, \kappa}(\gamma)}-F(f, g)\right| \leq 2\|f\|_{\mathcal{C}^{p, \kappa}(\gamma)}\|g\|_{L^{\infty}(\gamma)}$.

\section{$\S 4$. Proofs of Theorems 1.1 and 1.2}

The main aim of this section is to prove Theorems 1.1 and 1.2 by using the lemmas established in the previous sections.

Proof of Theorem 1.1. Let $p \in[1, \infty)$, and let $\kappa \in(0,1] \cap(0, p)$. If $g \in$ $L^{\infty}(\gamma)$, then for any $f \in \mathcal{M}^{p, \kappa}(\gamma)$, it is obvious that $f g \in \mathcal{M}^{p, \kappa}(\gamma)$ and $\|f g\|_{\mathcal{M}^{p, \kappa}(\gamma)} \leq\|g\|_{L^{\infty}(\gamma)}\|f\|_{\mathcal{M}^{p, \kappa}(\gamma)}$.

Conversely, suppose that $g$ is a pointwise multiplier on $\mathcal{M}^{p, \kappa}(\gamma)$. Then, for any $B \in \mathcal{B}_{1}$, letting $f$ (associated to $B$ ) be the function defined in Lemma 3.3, we apply Lemma 3.3(iii) to conclude that

$$
\begin{aligned}
{\left[\frac{1}{\gamma(B)} \int_{B}|g(x)|^{p} d \gamma(x)\right]^{1 / p} } & \lesssim\left[\frac{1}{\gamma(B)} \int_{B}|f(x) g(x)|^{p} d \gamma(x)\right]^{1 / p} \\
& \lesssim\|f g\|_{\mathcal{M}^{p, \kappa}(\gamma)} \lesssim\|g\|_{\mathcal{M}^{p, \kappa}(\gamma) \rightarrow \mathcal{M}^{p, \kappa}(\gamma)}
\end{aligned}
$$


Further, the differential theorem of integrals implies that $\|g\|_{L^{\infty}(\gamma)} \lesssim$ $\|g\|_{\mathcal{M}^{p, \kappa}(\gamma) \rightarrow \mathcal{M}^{p, \kappa}(\gamma)}$. This concludes the proof of Theorem 1.1.

Proof of Theorem 1.2. To show the sufficiency, we need to prove that, for any functions $g \in L^{\infty}(\gamma) \cap \mathcal{L}_{\Psi_{\kappa}}^{p, \kappa}(\gamma)$ and $f \in \mathcal{L}^{p, \kappa}(\gamma)$,

$$
\|g f\|_{\mathcal{L}^{p, \kappa}(\gamma)} \lesssim\|f\|_{\mathcal{L}^{p, \kappa}(\gamma)}\left[\|g\|_{L^{\infty}(\gamma)}+\|g\|_{\mathcal{L}_{\Psi \kappa}^{p, \kappa}(\gamma)}\right]
$$

Indeed, it is obvious that $\|g f\|_{L^{1}(\gamma)} \leq\|f\|_{L^{1}(\gamma)}\|g\|_{L^{\infty}(\gamma)}$. For all balls $B \in \mathcal{B}_{1}$, applying Lemma 3.2 and the fact that $\Psi_{\kappa}(B)[\gamma(B)]^{-\kappa / p} \gtrsim 1$, we have

$$
\begin{aligned}
& \left|f_{B}\right|\left[\frac{1}{[\gamma(B)]^{1-\kappa}} \int_{B}\left|g(x)-g_{B}\right|^{p} d \gamma(x)\right]^{1 / p} \\
& \quad \lesssim\|f\|_{\mathcal{L}^{p, \kappa}(\gamma)} \Psi_{\kappa}(B)\left[\frac{1}{\gamma(B)} \int_{B}\left|g(x)-g_{B}\right|^{p} d \gamma(x)\right]^{1 / p} \\
& \quad \lesssim\|f\|_{\mathcal{L}^{p, \kappa}(\gamma)}\|g\|_{\mathcal{L}_{\Psi_{\kappa}}^{p, \kappa}(\gamma)} .
\end{aligned}
$$

From this and Lemma 3.5, it follows that

$$
\|f g\|_{\mathcal{C}^{p, \kappa}(\gamma)} \lesssim\|f\|_{\mathcal{L}^{p, \kappa}(\gamma)}\left[\|g\|_{L^{\infty}(\gamma)}+\|g\|_{\mathcal{L}_{\Psi_{\kappa}}^{p, \kappa}(\gamma)}\right]
$$

Therefore, (4.1) holds, which proves the sufficiency part.

Now we show the necessary part for the case $\kappa \in[-p / n, 0)$. For any $B \in \mathcal{B}_{1}$, let $f$ be the function (associated to $B$ ) defined as in Lemma 3.3. With such an $f$, by Lemmas $3.3(\mathrm{i})$ and 3.2 and the fact that $\Psi_{\kappa}(B) \gtrsim 1$, we conclude that

$$
\begin{aligned}
& {\left[\frac{1}{\gamma(B)} \int_{B}|g(x)|^{p} d \gamma(x)\right]^{1 / p}} \\
& \quad \lesssim \frac{1}{\Psi_{\kappa}(B)}\left[\frac{1}{[\gamma(B)]^{1-\kappa}} \int_{B}|f(x) g(x)|^{p} d \gamma(x)\right]^{1 / p} \\
& \quad \lesssim\left[\frac{1}{[\gamma(B)]^{1-\kappa}} \int_{B}\left|f(x) g(x)-(f g)_{B}\right|^{p} d \gamma(x)\right]^{1 / p}+\frac{\left|(f g)_{B}\right|}{\Psi_{\kappa}(B)[\gamma(B)]^{-\kappa / p}} \\
& \quad \lesssim\|f g\|_{\mathcal{L}^{p, \kappa}(\gamma)} \lesssim\|g\|_{\mathcal{L}^{p, \kappa}(\gamma) \rightarrow \mathcal{L}^{p, \kappa}(\gamma)} .
\end{aligned}
$$

From this and the differential theorem of integrals, it follows that $g \in L^{\infty}(\gamma)$ and that

$$
\|g\|_{L^{\infty}(\gamma)} \lesssim\|g\|_{\mathcal{L}^{p, \kappa}(\gamma) \rightarrow \mathcal{L}^{p, \kappa}(\gamma)}
$$


Moreover, for any $B \in \mathcal{B}_{1}$, with $f$ as in Lemma 3.3 , since $f, f g \in \mathcal{L}^{p, \kappa}(\gamma)$ and $g \in L^{\infty}(\gamma)$, we apply Lemmas 3.3(i) and 3.5 to conclude that

$$
\begin{aligned}
& \Psi_{\kappa}(B)\left[\frac{1}{\gamma(B)} \int_{B}\left|g(x)-g_{B}\right|^{p} d \gamma(x)\right]^{1 / p} \\
& \quad \lesssim\left|f_{B}\right|\left[\frac{1}{[\gamma(B)]^{1-\kappa}} \int_{B}\left|g(x)-g_{B}\right|^{p} d \gamma(x)\right]^{1 / p} \\
& \quad \lesssim\|f g\|_{\mathcal{L}^{p, \kappa}(\gamma)}+\|f\|_{\mathcal{L}^{p, \kappa}(\gamma)}\|g\|_{L^{\infty}(\gamma)} \\
& \quad \lesssim\|g\|_{\mathcal{L}^{p, \kappa}(\gamma) \rightarrow \mathcal{L}^{p, \kappa}(\gamma)} .
\end{aligned}
$$

Then, taking the supremum over all $B \in \mathcal{B}_{1}$, we see that

$$
\|g\|_{\mathcal{L}_{\Psi_{\kappa}}^{p, \kappa}(\gamma)} \lesssim\|g\|_{\mathcal{L}^{p, \kappa}(\gamma) \rightarrow \mathcal{L}^{p, \kappa}(\gamma)}
$$

Combining (4.2) and (4.3) implies that $g \in L^{\infty}(\gamma) \cap \mathcal{L}_{\Psi_{\kappa}}^{p, \kappa}(\gamma)$ and hence proves the necessary part for the case $\kappa \in[-p / n, 0)$.

To prove the necessary part for the case $\kappa=0$, we notice that the above arguments remain valid if we replace the function $f$ therein with the function $h$ as in Lemma 3.4. The details are omitted. This concludes the proof of Theorem 1.2.

\section{$\S 5$. Some examples}

The main aim of this section is to present some examples of the class of pointwise multipliers on $\operatorname{BMO}(\gamma)$. First, we apply Remark 1.2(ii) to construct a function which is a pointwise multiplier on $\mathrm{BMO}(\gamma)$ but has an oscillation at infinity.

ExAmple 5.1. Let $f(x):=\sin \ln (1+|x|)$ for all $x \in \mathbb{R}^{n}$. Then, $f$ is a pointwise multiplier on $\operatorname{BMO}(\gamma)$.

Proof. Clearly, $f \in L^{\infty}(\gamma)$. For all $B \in \mathcal{B}_{1}$ and $x, y \in B$, by the mean value theorem, we have

$$
\begin{aligned}
|f(x)-f(y)| & =|\sin \ln (1+|x|)-\sin \ln (1+|y|)| \\
& \leq \sup _{\xi \in B} \frac{|\cos \ln (1+|\xi|)||x-y|}{1+|\xi|} \lesssim \frac{r_{B}}{1+\left|c_{B}\right|},
\end{aligned}
$$

where the last step holds because $1+|\xi| \sim 1+\left|c_{B}\right|$ for all $\xi \in B \in \mathcal{B}_{1}$. Then, from $r_{B}\left|c_{B}\right| \leq 1$ and $r_{B} \ln \left(2 / r_{B}\right) \lesssim 1$, it follows that, for all $B \in \mathcal{B}_{1}$ and 
$x, y \in B$,

$$
|f(x)-f(y)|\left[\left|c_{B}\right|^{2}+\ln \frac{2}{r_{B}}\right] \lesssim 1,
$$

which, combined with Remark 1.2(ii), implies that $f$ is a pointwise multiplier on $\operatorname{BMO}(\gamma)$.

The next example shows that a function with polynomial decay at infinity of order greater than 1 is a pointwise multiplier on $\operatorname{BMO}(\gamma)$.

EXAMPLE 5.2. Let $\phi$ be a function supported on $\left\{x \in \mathbb{R}^{n}:|x| \geq 3 / 2\right\}$ such that $\phi, \nabla \phi \in L^{\infty}(\gamma)$. For any given $\alpha \geq 1$, define $g_{\alpha}(x):=\phi(x) /|x|^{\alpha}$ for all $x \in \mathbb{R}^{n}$. Then $g_{\alpha}$ is a pointwise multiplier on $\operatorname{BMO}(\gamma)$.

Proof. By the support condition of $\phi$, we know that $\left\|g_{\alpha}\right\|_{L^{\infty}(\gamma)} \lesssim$ $\|\phi\|_{L^{\infty}(\gamma)}$. Fix $B \in \mathcal{B}_{1}$, and fix $x, y \in B$. Again, by the support condition of $\phi$, we see that if $\left|g_{\alpha}(x)-g_{\alpha}(y)\right| \neq 0$, then either $|x| \geq 3 / 2$ or $|y| \geq 3 / 2$ and that, whatever the case, it holds that $1 / 2<\left|c_{B}\right| \sim|x| \sim|y|$. Hence, by the mean value theorem, we have

$$
\begin{aligned}
\left|g_{\alpha}(x)-g_{\alpha}(y)\right| & \leq \frac{|\phi(x)-\phi(y)|}{|x|^{\alpha}}+|\phi(y)| \mid \frac{1}{|x|^{\alpha}}-\frac{1}{|y|^{\alpha} \mid} \\
& \leq \frac{\|\nabla \phi\|_{L^{\infty}(\gamma)}|x-y|}{|x|^{\alpha}}+\|\phi\|_{L^{\infty}(\gamma)} \frac{\left.|| x\right|^{\alpha}-|y|^{\alpha} \mid}{|x|^{\alpha}|y|^{\alpha}} \lesssim \frac{r_{B}}{\left|c_{B}\right|^{\alpha}} .
\end{aligned}
$$

Further, by $\alpha \geq 1,\left|c_{B}\right|>1 / 2, r_{B} \leq 1$, and $r_{B}\left|c_{B}\right| \leq 1$, we see that then $\left(r_{B} /\left|c_{B}\right|^{\alpha}\right)\left[\left|c_{B}\right|^{2}+\ln \left(2 / r_{B}\right)\right] \lesssim 1$. This and Remark 1.2(ii) imply that $g_{\alpha}$ is a pointwise multiplier on $\operatorname{BMO}(\gamma)$.

The following example is partly motivated by [30], which is needed for constructing a nonnegative function that lies in $\operatorname{BMO}(\gamma)$ but not in $\operatorname{BLO}(\gamma)$ in Example 5.4 below.

ExAmple 5.3. For all $x \in \mathbb{R}^{n}$, define

$$
g(x):=\int_{\min \{1,|x|\}}^{2} \frac{1}{\ln \frac{2}{\min \{1, t\}}} \frac{d t}{t} .
$$

Then $g, \sin g, \cos g \in \mathrm{BMO}^{\log }(\gamma)$ and hence are pointwise multipliers on $\operatorname{BMO}(\gamma)$. 
Proof. Notice that $g(x)=1-\ln \ln 2+\ln \ln (2 /|x|)$ when $|x|<1$ and that $g(x)=1$ when $|x| \geq 1$.

First we show that $g \in \mathrm{BMO}^{\log }\left(\mathbb{R}^{n}\right)$. If $B \in \mathcal{B}_{1}$ with $\left|c_{B}\right| \geq 2$, then $g(x)=1$ for all $x \in B$, and consequently, $(1 / \gamma(B)) \int_{B}\left|g(x)-g_{B}\right| d \gamma(x)=0$. If $\left|c_{B}\right|<2$, then $\left|c_{B}\right|^{2}+\ln \left(2 / r_{B}\right) \sim \ln \left(2 / r_{B}\right)$ by using $\ln \left(2 / r_{B}\right) \geq \ln 2$. Thus, to show that $g \in \mathrm{BMO}^{\log }(\gamma)$, we need only to prove that

$$
\sup _{B \in \mathcal{B}_{1},\left|c_{B}\right|<2} \frac{\ln \frac{2}{r_{B}}}{\gamma(B)} \int_{B}\left|g(x)-g_{B}\right| d \gamma(x)<\infty .
$$

Consider the ball $B \in \mathcal{B}_{1}$ such that $\left|c_{B}\right|<2$. Observing that, by (1.3),

$$
\frac{1}{\gamma(B)} \int_{B}\left|g(x)-g_{B}\right| d \gamma(x) \sim \frac{1}{|B|} \int_{B}\left|g(x)-g_{B}^{L}\right| d x,
$$

where $g_{B}^{L}:=(1 /|B|) \int_{B} g(y) d y$, we see that (5.1) holds, provided that

$$
\sup _{\substack{B \in \mathcal{B}_{1},\left|c_{B}\right|<2 \\ B \cap B(0,1) \neq \emptyset}} \frac{\ln \frac{2}{r_{B}}}{|B|} \int_{B}\left|g(x)-g_{B}^{L}\right| d x<\infty .
$$

Because (5.3) was implicitly proved in [36, Theorem 2] (see also [30, Lemma 2.4]), $g \in \mathrm{BMO}^{\log }(\gamma)$.

Noticing that $|\sin a-\sin b| \leq|a-b|$ for all $a, b \in \mathbb{R}$, we easily see that

$$
\frac{1}{\gamma(B)} \int_{B}\left|\sin g(x)-(\sin g)_{B}\right| d \gamma(x) \leq \frac{2}{\gamma(B)} \int_{B}\left|g(x)-g_{B}\right| d \gamma(x) .
$$

From this and $g \in \mathrm{BMO}^{\log }\left(\mathbb{R}^{n}\right)$, it follows that $\sin g \in \mathrm{BMO}^{\log }(\gamma)$. Likewise, we have $\cos g \in \mathrm{BMO}^{\log }(\gamma)$. This finishes the proof.

Recall that the space $\mathrm{BLO}(\gamma)$ on the Gauss measure space was introduced in [19] and the boundedness of maximal singular integrals from $L^{\infty}(\gamma)$ to $\operatorname{BLO}(\gamma)$ was also obtained. A locally integrable function $f$ is said to be in the space $\mathrm{BLO}(\gamma)$ if

$$
\|f\|_{\mathrm{BLO}(\gamma)}:=\|f\|_{L^{1}(\gamma)}+\sup _{B \in \mathcal{B}_{1}}\left[\frac{1}{\gamma(B)} \int_{B} f(y) d \gamma(y)-\underset{x \in B}{\operatorname{essinf}} f(x)\right]<\infty .
$$

It was proved in $[19$, Remark 2.1(iii)] that $\mathrm{BLO}(\gamma)$ is a proper subspace of $\operatorname{BMO}(\gamma)$. The following example further shows that nonnegative functions in $\operatorname{BMO}(\gamma)$ may not belong to $\operatorname{BLO}(\gamma)$. This tells us that the maximal singular integral operators map $L^{\infty}(\gamma)$ to $\{f \in \operatorname{BLO}(\gamma): f \geq 0\}$ is strictly contained in $\operatorname{BMO}(\gamma)$ by noting that they are nonnegative. 
ExAmple 5.4. Let $g$ be the function defined in Example 5.3. Consider $n=1$, and define $f(x):=\left|\sin g(x) \ln (2 /|x|) \chi_{|x| \leq 2}(x)\right|$ for all $x \in \mathbb{R}$. Then, $f \in \operatorname{BMO}(\gamma)$, but $f \notin \operatorname{BLO}(\gamma)$.

Proof. Let $h(x):=\ln (2 /|x|) \chi_{|x| \leq 2}(x)$ for all $x \in \mathbb{R}$. It is easy to see that $h \in \operatorname{BMO}(\gamma)$ by $(5.2)$ and that $h \in \operatorname{BMO}(\mathbb{R})$. From Example 5.3, we deduce that $\sin g$ is a pointwise multiplier on $\operatorname{BMO}(\gamma)$, and hence $f \in \operatorname{BMO}(\gamma)$ by using Theorem 1.2.

Next we show that $f \notin \operatorname{BLO}(\gamma)$. In Example 5.3, it was proved that when $|x|<1, g(x)=1-\ln \ln 2+\ln \ln (2 /|x|)$, which is strictly decreasing and positive on $\left(0, e^{-1}\right)$. Thus, we choose a strictly decreasing sequence $\left\{r_{k}\right\}_{k=10}^{\infty} \subset(0,1)$ such that $(\pi / 4) k=g\left(r_{k}\right)$ and $\lim _{k \rightarrow \infty} r_{k}=0$. Based on the proof of [17, Proposition 2.1], we see that, for each $m \in \mathbb{N}$,

$$
\begin{aligned}
& \frac{1}{r_{8 m+3}-r_{8 m+4}} \int_{\left(r_{8 m+4}, r_{8 m+3}\right)}\left[f(x)-\underset{y \in\left(r_{8 m+4}, r_{8 m+3}\right)}{\operatorname{essinf}} f(y)\right] d x \\
& \geq \frac{1}{2} \frac{\sqrt{2} \ln \left(2 / r_{8 m+3}\right)}{2},
\end{aligned}
$$

which tends to $\infty$ as $m \rightarrow \infty$. Notice that all the intervals $\left(r_{8 m+4}, r_{8 m+3}\right)$ belong to the admissible class $\mathcal{B}_{1}$ and that, for any $m \in \mathbb{N}$,

$$
\begin{gathered}
\frac{1}{\gamma\left(\left(r_{8 m+4}, r_{8 m+3}\right)\right)} \int_{\left(r_{8 m+4}, r_{8 m+3}\right)}\left[f(x)-\operatorname{essinf}_{y \in\left(r_{8 m+4}, r_{8 m+3}\right)}^{\operatorname{ess}} f(y)\right] d \gamma(x) \\
\sim \frac{1}{r_{8 m+3}-r_{8 m+4}} \int_{\left(r_{8 m+4}, r_{8 m+3}\right)}\left[f(x)-\underset{y \in\left(r_{8 m+4}, r_{8 m+3}\right)}{\operatorname{essinf}} f(y)\right] d x .
\end{gathered}
$$

Therefore, $f \notin \operatorname{BLO}(\gamma)$. This finishes the proof of Example 5.4.

Acknowledgments. The authors are very grateful to Professor Peter Sjögren for his valuable remarks on this manuscript. They also wish to thank the referees for many constructive and helpful comments which essentially improved our exposition.

\section{REFERENCES}

[1] A. Carbonaro, G. Mauceri, and S. Meda, $H^{1}$ and BMO for certain locally doubling metric measure spaces, Ann. Sc. Norm. Super. Pisa Cl. Sci. (5) 8 (2009), 543-582. MR 2581426.

[2] - $H^{1}$ and BMO for certain locally doubling metric measure spaces of finite measure, Colloq. Math. 118 (2010), 13-41. MR 2600517. DOI 10.4064/cm118-1-2. 
[3] R. R. Coifman and G. Weiss, Analyse harmonique non-commutative sur certains espaces homogènes, Lecture Notes in Math. 242, Springer, Berlin, 1971. MR 0499948.

[4] - Extensions of Hardy spaces and their use in analysis, Bull. Amer. Math. Soc. (N.S.) 83 (1977), 569-645. MR 0447954.

[5] L. Diening, Maximal function on Musielak-Orlicz spaces and generalized Lebesgue spaces, Bull. Sci. Math. 129 (2005), 657-700. MR 2166733. DOI 10.1016/j.bulsci. 2003.10.003.

[6] E. B. Fabes, C. E. Gutiérrez, and R. Scotto, Weak-type estimates for the Riesz transforms associated with the Gaussian measure, Rev. Mat. Iberoam. 10 (1994), 229-281. MR 1286476. DOI 10.4171/RMI/152.

[7] L. Forzani and R. Scotto, The higher order Riesz transform for Gaussian measure need not be weak type (1,1), Studia Math. 131 (1998), 205-214. MR 1644460.

[8] J. García-Cuerva, G. Mauceri, P. Sjögren, and J. L. Torrea, Higher-order Riesz operators for the Ornstein-Uhlenbeck semigroup, Potential Anal. 10 (1999), 379-407. MR 1698617. DOI 10.1023/A:1008685801945.

[9] - Spectral multipliers for the Ornstein-Uhlenbeck semigroup, J. Anal. Math. 78 (1999), 281-305. MR 1714425. DOI 10.1007/BF02791138.

[10] C. E. Gutiérrez, On the Riesz transforms for Gaussian measures, J. Funct. Anal. 120 (1994), 107-134. MR 1262249. DOI 10.1006/jfan.1994.1026.

[11] C. E. Gutiérrez, C. Segovia, and J. L. Torrea, On higher order Riesz transforms for Gaussian measures, J. Fourier Anal. Appl. 2 (1996), 583-596. MR 1423529. DOI 10. 1007/s00041-001-4044-1.

[12] E. Harboure, J. L. Torrea, and B. Viviani, Vector-valued extensions of operators related to the Ornstein-Uhlenbeck semigroup, J. Anal. Math. 91 (2003), 1-29. MR 2037400. DOI 10.1007/BF02788780.

[13] A. Hartmann, Pointwise multipliers in Hardy-Orlicz spaces, and interpolation, Math. Scand. 106 (2010), 107-140. MR 2603465.

[14] S. Janson, On functions with conditions on the mean oscillation, Ark. Mat. 14 (1976), 189-196. MR 0438030.

[15] L. D. Ky, New Hardy spaces of Musielak-Orlicz type and boundedness of sublinear operators, Integral Equations Operator Theory 78 (2014), 115-150. MR 3147406. DOI 10.1007/s00020-013-2111-z.

[16] A. K. Lerner, Some remarks on the Hardy-Littlewood maximal function on variable $L^{p}$ spaces, Math. Z. 251 (2005), 509-521. MR 2190341. DOI 10.1007/ s00209-005-0818-5.

[17] H. Lin, E. Nakai, and D. Yang, Boundedness of Lusin-area and $g_{\lambda}^{*}$ functions on localized BMO spaces over doubling metric measure spaces, Bull. Sci. Math. 135 (2011), 59-88. MR 2764953. DOI 10.1016/j.bulsci.2010.03.004.

[18] L. Liu, Y. Sawano, and D. Yang, Morrey-type spaces on the Gauss measure spaces and boundedness of singular integrals, J. Geom. Anal., published electronically 2 October 2012. DOI 10.1007/s12220-012-9362-9.

[19] L. Liu and D. Yang, BLO spaces associated with the Ornstein-Uhlenbeck operator, Bull. Sci. Math. 132 (2008), 633-649. MR 2474485. DOI 10.1016/j.bulsci.2008.08. 003.

[20] J. Maas, J. van Neerven, and P. Portal, Conical square functions and non-tangential maximal functions with respect to the Gaussian measure, Publ. Mat. 55 (2011), 313341. MR 2839445. DOI 10.5565/PUBLMAT_55211_03. 
[21] - Whitney coverings and the tent spaces $T^{1, q}(\gamma)$ for the Gaussian measure, Ark. Mat. 50 (2012), 379-395. MR 2961328. DOI 10.1007/s11512-010-0143-z.

[22] L. Maligranda and E. Nakai, Pointwise multipliers of Orlicz spaces, Arch. Math. (Basel) 95 (2010), 251-256. MR 2719383. DOI 10.1007/s00013-010-0160-y.

[23] G. Mauceri and S. Meda, BMO and $H^{1}$ for the Ornstein-Uhlenbeck operator, J. Funct. Anal. 252 (2007), 278-313. MR 2357358. DOI 10.1016/j.jfa.2007.06.017.

[24] G. Mauceri, S. Meda, and P. Sjögren, Endpoint estimates for first-order Riesz transforms associated to the Ornstein-Uhlenbeck operator, Rev. Mat. Iberoam. 28 (2012), 77-91. MR 2904131. DOI 10.4171/RMI/667.

[25] - A maximal function characterization of the Hardy space for the Gauss measure, Proc. Amer. Math. Soc. 141 (2013), 1679-1692. MR 3020855. DOI 10.1090/ S0002-9939-2012-11443-1.

[26] T. Menárguez, S. Pérez, and F. Soria, The Mehler maximal function: A geometric proof of the weak type 1, J. Lond. Math. Soc. (2) 61 (2000), 846-856. MR 1766109. DOI 10.1112/S0024610700008723.

[27] E. Nakai, Pointwise multipliers for functions of weighted bounded mean oscillation, Studia Math. 105 (1993), 105-119. MR 1226621.

[28] - Pointwise multipliers on weighted BMO spaces, Studia Math. 125 (1997), 35-56. MR 1455621.

[29] - A characterization of pointwise multipliers on the Morrey spaces, Sci. Math. Jpn. 3 (2000), 445-454. MR 1803059.

[30] E. Nakai and K. Yabuta, Pointwise multipliers for functions of bounded mean oscillation, J. Math. Soc. Japan 37 (1985), 207-218. MR 0780660. DOI 10.2969/jmsj/ 03720207.

[31] , Pointwise multipliers for functions of weighted bounded mean oscillation on spaces of homogeneous type, Sci. Math. Japon. 46 (1997), 15-28. MR 1466111.

[32] S. Pérez, The local part and the strong type for operators related to the Gaussian measure, J. Geom. Anal. 11 (2001), 491-507. MR 1857854. DOI 10.1007/BF02922016.

[33] G. Pisier, "Riesz transforms: A simpler analytic proof of P.-A. Meyer's inequality" in Séminaire de probabilités, XXII, Lecture Notes in Math. 1321, Springer, Berlin, 1988, 485-501. MR 0960544. DOI 10.1007/BFb0084154.

[34] P. Sjögren, "On the maximal function for the Mehler kernel" in Harmonic Analysis (Cortona, 1982), Lecture Notes in Math. 992, Springer, Berlin, 1983, 73-82. MR 0729346. DOI 10.1007/BFb0069151.

[35] - Operators associated with the Hermite semigroup - a survey, J. Fourier Anal. Appl. 3 (1997), 813-823. MR 1600191. DOI 10.1007/BF02656487.

[36] S. Spanne, Some function spaces defined using the mean oscillation over cubes, Ann. Sc. Norm. Super. Pisa (3) 19 (1965), 593-608. MR 0190729.

[37] D. A. Stegenga, Bounded Toeplitz operators on $H^{1}$ and applications of the duality between $H^{1}$ and the functions of bounded mean oscillation, Amer. J. Math. 98 (1976), 573-589. MR 0420326 .

[38] W. Urbina, On singular integrals with respect to the Gaussian measure, Ann. Sc. Norm. Super. Pisa Cl. Sci. (4) 17 (1990), 531-567. MR 1093708.

[39] K. Yabuta, Pointwise multipliers of weighted BMO spaces, Proc. Amer. Math. Soc. 117 (1993), 737-744. MR 1123671. DOI 10.2307/2159136. 
Liguang Liu

Department of Mathematics

School of Information

Renmin University of China

Beijing 100872

People's Republic of China

liuliguang@ruc.edu.cn

Dachun Yang (corresponding author)

School of Mathematical Sciences

Beijing Normal University

Laboratory of Mathematics and Complex Systems

Ministry of Education

Beijing 100875

People's Republic of China

dcyang@bnu.edu.cn 